CASOS CLINICOS

Rev. Chil. Pedietr. 58(3): 233-236, 1987

\title{
Síndrome de muerte súbita infantil (SMSI): tres casos de monitoría en domicilio
}

\author{
Dr. Osvaldo Artaza B. ${ }^{1}$; Dr. Jaime Cordero T. ${ }^{2}$; E.U. Carmen Hernández $\mathrm{F}^{3}{ }^{3}$ \\ Sudden infant death syndrome (SIDS) home monitoring
}

\begin{abstract}
Three cases of infants at risk of sudden death, that were monitored at home, ate presented. One of tle patients lod two siblings that died by unexplained reasons and he suffered, at 2 month of agc, an episodc of near-miss SIDS. During monitoring he had thrce alarns that responded to stimulus. After 9 months of age monitoring was stoped uneventfully. The second infant had 6 siblings that died unexpectediy when between 2 and 5 months old, without apparent causes: at 2 months of age he had a near-miss SIDS, and 2 months later hedied, even though the alarm went on and emergency measures were taken. The third case is that of an infant monitored from the ago of two months because a brotler died suddenly: there were no alams along 4 months of monitoring at home. It is important to statilish criterias to monitor infant at risk of sudden death. It is still too early to make conclusions over the eflectivity of monitoring at home; even though there is no evidence that monitoring decreases SIDS mortality. it should still be used among infants that have had episodes of near-miss SIDS, specially if a sibling had died and there exist a high level of anxiety in the family.

(Key words: sudden infant death syndrome, SIDS, apnea monitor, home monitoring).
\end{abstract}

La muerte inesperada e inexplicable en los lactantes continúa siendo, un dificil problema clínico $^{1,8}$. El dramatismo de estas muertes es de conocimiento público a través de los medios de comunicación social, por lo que existe un alto grado de sensibilidad ante este problema. Los padres que han tenido la experiencia del fallecimiento de uno de sus bijos, enfrentan con gran ansiedad un segundo niño $y$ guardan grandes esperanzas en que un monitor de apnea (MA) evite otrá muerte. La hipótesis de que los niños que fallecieron por SMSI tuvieron detención de sus movimientos respiratorios, de probablemente más de 30 segundos antes de morir o de sufrir daño itreversible ${ }^{9}$, no puedo ser comprobada yá que desconocemos como ellos mueren realmente. A pesar de esto, aún se acepta que la interrupción de la ventilación puede preceder al dano irrecuperable, y que por lo tanto, un MA podria evitar la muerte permitiendo est imulos o maniobras de reanimación oportunas. Sin embargo, hay casos bicn documentados de muertes en niños vigilados a pesar de haberse realizado las maniobras adecuradas inmediatamente después del sonido de la alarma ${ }^{10}$, lo que sugiere que ef $\mathrm{MA}$ no garantiza

1. Médico becario, servicio de pediatria, Hospital Luis Cało Mackenna.

2. Médico jefe unidad cuidados intensivos. Hospital Luis Calvo Mackenna.

3. Enfermera jefe unidad cuidados intensivos. Lospital Luis Calyo Mackenna. que se evite la muerte. Por otra parte, muchos padres afirman que sin el MA sus hijos habrían fallecido ${ }^{11}$. El uso de MA en domicilio, es un tema polémico ${ }^{9-13}, 15-17$, tiene elevado costo, produce falsas alarmas y falla en la detección de ciertas apneas ${ }^{9}$.

Con el propósito de evaluar la eficacia e ilustrar aspectos relevantes de la monitoría en domicilio, se presentan tres pacientes que han sido sometidos a ella como parte de un programa de base hospitalaria.

\section{Casos clínicos}

1. Nitio varón eutrófico, examen físico y neurológico normal, dos hermanos fallecieron a los 2 y 3 meses de edad sin causa aparente. Parto eutócico, a las 38 semanas de gestación; peso de nacimiento $2.850 \mathrm{~g}$ : período perinatal normal. Alimentación con lactancia materna exclusiva. Los padres se turraban para permanecer despier. tos durante la noche. A los 2 meses de edad sufrió un episodio de apnea, hipotonía y cianosis durante el sueño que responđió a estímulos de la madre. Observado durante 14 días en el hospital no presentó problemas: hemograma, orina, glicemia, creatininemia, gases arteriales, electrolitos plasmáticos, electrocardiograma, radiografía de tórax, radiografía de trátsito esofágico y electrocncefalograma normales. El neumucardiograma no mostró alteraciones en un registro de $24 \mathrm{~h}$. 
Vigilado con monitor de apnea en su domicilio, tuvo un promedio de 6 falsas alarmas semanales $y$, entre $\operatorname{los} 4$ y 5 meses de edad, 3 alarmas verdaderas, todas durante el sueño, que respondieron a estímulos, no siendo necesarias maniobras de resucitación. E] monitor se retiró a los 9 meses de edad. Actualmente está sano, tiene 1 año 5 meses.

2. Varón nacido por parto eutócico, pesando $3.080 \mathrm{~g}, \sin$ afecciones perinatales, lactancia materna exclusiva. Seis hermanos fallecieron entre los 2 y 5 meses de edad, todos eran lactantes eutróficos, tres de sexo masculino, aparentemente sanos, sin patologia perinatal y murieron durante el sueño sin explicación. En ninguno se realizó autopsia. A los dos meses de edad el paciente sufrió un episodio de apnea y cianosis intensa que cedió con estimulos de sus padres; su examen físico era normal y en un estudio similar al descrito en el caso anterior sólo se encontró reflujo gastro-esofágico moderado que fue tratado con medicamentos. Se indicó vigilancia de apnea en domicilio, registrándose en promedio 8 falsas alarmas semonales. Falleció durante el sueño a los 4 meses de edad, estando aparentemente sano, a pesar de que el monitor dio aviso y se realizaron maniobras de reanimación inmediatos (no se practicó autopsia por deseo de sus padres).

3. Niño de sexo masculino, nacido en parto cutócico, pesó al nacer $4.230 \mathrm{~g}$ no tuvo problemas perinatales. Su hermano anterior murió súbitamente a $\operatorname{los} 3$ meses de edad, sin que en la autopsia se identificase la causa de muerte. $\Lambda 1$ nes de edad se realizaron exámenes similares a los casos anteriores, los que resultaron nomales, excepto radiografías esofágicas que mostraron reflujo gastro-esofágico leve, actualmente en tratamiento médico. Su evolución pondo-estatural y sicomotor han sido adecuados. Se mantiene con monitoría en su domicilio, con un promedio de 3 falsas alarmas semanales y ninguna verdadera después de cuatro meses de vigilancia electrónica de la spnea.

\section{COMENTARIO}

En el primer caso descrito, las muertes de los hermanos son altamente sospechosas de SMSI, pero no confirmadas por no haber sido sometidos a autopsia. En este niño parece beneficioso el empleo de monitoría en el domicilio, ya que las tres alarmas verdaderas estuvieron bien documentadas, aunque nunca se podrá saber cual habria sido la evolución espontánea de este niño, de no mediar el aviso del monitor y el estínulo oportuno de sus padres, quienes "al fin pudieren dormir tranquilos". La tolerancia de la monitoría fue excclente a pesar de numerosas falsas alarmas, incluso se produjo un excesivo apego al $\mathrm{MA}$ haciendose dificil su retiro.

En el segundo caso el programa no contribuyó a evitar la muerte (altamente sospechosa de SMSI). Este hecho nos efectó profundamente, nos obligó revisar nuestro programa de instrucción en técnicas de reanimación, a pesar de que los padres relataron haber seguido bien las instrucciones. Estos se habían formado grandes expectativas de que el monitor evitaría la muerte. El tercer caso nos deja dudas, difíciles de aclarar, sobre la justifjcación de la monitoría. En el diseño de un programa de monitoría en domicilio, purece fundamental considerar, entre varios aspectos siguientes:

Es necesario contar con un sistema de control de la ventilacion, que identifique correctamente el cese de la respiración con cl minimo de falsas alarmas. Vosotros hemos usado un MA Graseby MR 10, que indica los movimientos respiratorios con sefiales sonoras y luminosas. La alarma se prefija para 10 ó 20 segundos de apnea. Se adhiere una cápsula llena de aire a la piel del abdomen con tela adhesiva $y$ al moverse la superficie abdominal por la expansión torácica se produce un cambio de presión que es transmitido por un tubo plástico hasta el monitor portátil, operando por baterías. Las falsas alarmas son frecuentes, generalmente debidas a problemas en la fijación de la cápsula. Como no dispone de alarma de bradicardia este modelo puede no advertir oportunamente una apnea obstructiva.

Se requicre, además, conocer los posibles efecros de la monitoria: fuera del al to costo, hay farnilias a las que se les hace francamente intolerable vivir bajo la constante tensión de las falsas alarmas ${ }^{13}$. En nuestra experiencia el MA ha reducido el nivel de ansiedad, expresando los padres que pueden dormir "confiados en el mo. nitor" y con la sensación de estar haciendo "todo lo posible" por su hijo.

Debe haber una adecuada selección de niños en riesgo: Los hermanos de niños fallecidos por SMSI tendrán un riesgo levemente superior al de la publación general, pero en todo caso menor al $1 \%$. siendo la incidencia general de 2 por mil nacidos vivos ${ }^{8}, 12,14,18,19$. Los niños de baja peso de nacimiento tienen más riesgo $14,20-21$ y los que han presentado episodios de casi-SMSI también ${ }^{9}$. No existen estudios que permitan precisar categóricamente que riesgo tiene un niño de fallecer por SMSI ${ }^{15-16,23}$ lo que obliga a revisar detenidamente cađa caso en particular, antes de decidir su inclusión en un programa de monitoria.

Los pad̀res de los niños seleccionados deben 
ser instruidos en el manejo del monitor; la identificación de las alarmas verdaderas, las técnicas de reanimación; el manejo de un registro escrito de alarmas en relación con síntomas y actividad del niño, junto con las conductas realizadas por lus padres en cada alarma y sobre "qué y cómo hacer" cuando el niño no responde prontamente. Nuestro programa incluye, dependiendo de la familia, unas cinco sesiones (tardes) teóricoprácticas a cargo de un médico y de una enfermera de gran experiencia en reanimación. Es también fundamental el apoyo psicológico del grupo farniliar, advirtiendo que el monitor no garantiza totalmente la sobrevida, como de la alta probabilidad de que el niñu no preserı te jamás un problema y de que es posible que tengan que soportar innecesariamente a un "ruidoso nuevo mienbro" en la familia. Hay que definir un sistema de seguimiento y control periódico del nino ${ }^{17}$, la famolia $y$ el monitor.

Finalmence hay que definir un plazo de mo. nitoría: la gran mayoría de las muertes se producen entre los 2 y 3 meses de edad ${ }^{3}$ y el $90 \%$ ocurren antes de los primcros 8 meses de vida ${ }^{9}$ Por lo que hemos definido el término de la vigilancia electrónica de aptrea en 3 meses después de la edad en la que falieciú el hermano, a no mediar alarmas verdaderas; y 4 meses después de la última alarma verdadera.

\section{RESUMEN}

Se presentan tres cusos de monitoria clectrónica en el domicilio a niños con riesgo de muerte súbita, de un programa del Hospital Luis Calvo Mackenna. Un lactante aparentemente sano, sin enfermedades previas, con dos hermanos fallecjdos en forma inexplicable, que a los 2 meses de edad tuvo un episodio compatible con casi síndrome infantil de muerte súbita (SMSI). Durante la yigilancia tuvo tres alarmas verdaderas que respondieron a estímulos. Sc retiro al monitor a $\operatorname{los} 9$ meses de edad y actualmente estí sano. El segundo paciente tenía antecedentes de 6 hermanos fallecidos entre los 2 y 5 meses de edad sin causa aparente. sulrió un episodio sugerente do casi SMSI a los 2 meses y falleció a los 4 meses de edad a pesar de que el monitur dio atiso y se realizaron maniobras oportunas. Finalmente un tercer lactante que fue vigilado desde los 2 meses de edad porque su hermano habia fallecido en forma súbita, no tuvo alarmas verdaderas en 4 meses de vigilancia en domicilio. Es fundamental definir un criterio subre qué niños en riesgo de muerte súbita deberín ser vigilados. y dar instrucción, apoyo y seguimiento al grupo familiar. Nuestra experiencia es aún pequeña para emitir un juicio sobre la eficacia de la monitoría en domicilio, y aunque no hay cvidencia de que esta disminuya la mortalidad pot SMSI, debe continuar planteándose en niños que han tenido episodios de casi -SMSI. especialmente si existen untecedentes de hermanos fallecidos $y$ alto nivel de ansiedad en la familia.

\section{REFERENCIAS}

1. Zehal, B.H., Friedmon, S.B.: Sudden infant deattr syndrome and infantile apnea: Introduction. Pediatric Ann 1984 Ann 13: 188, 1984.

2. Voldés-Dapena, M.A.: Sudden infant death syudrome: A review of the nedical literaturc 1974-1979. Pediatrics 66: 597,1980 .

3. Shannon, D.C., Kelly, D.H.: SIUS und near-SIDS, $\therefore$ lingl J Yed 306: 959, 1982.

4. Kelly, D.H. Shannon, D.C.: Sudden jniant death symdrome and neat sudden infant duath syndrome: A review of the literature 1964-1982. Pediatr Clin North Am 29; 1241, 1982.

5. Brooks, J.G.: Aprea of intancy and sudden infant des th syndrome. Am J Dis Child 136:1012, 1982

6. Ariogno, R.t.: Fualuation and management of infantile apnea, Pediatrics Ant 13: 210, 1984.

7. Arianno, K.L., Guilleminault, C, Karobkin. R. et al: "Vear-miss" for sudden intant death syndtome: A clinical problem. Pediatrics 71: 726, 1983.

8. Brady, J.P., Gould. J.B.: Sudden infant death syndrome. The physucin's dilemma. Ady P'ediatr $30: 635,1983$.

9. Miner, A.D.: Apnoca monitors and sudden infant death: Report trum the Foundation lor the study of sudden iniant death and the British Pediatric Respiratury Group. Arcil Dis C'hild 60: 76, 1985.

10. Iewok, $N$.: Suducn inlant death syndrome in a hospitalised isfant on an apnota monitor. Pediatrics $56: 2696,1975$.

11. Kell, D.H., Shomnon, D.C., O'Connell, K.: rare of infants witl " "near-miss" sudden infant cleath syndrome. Pediatrics 61: $511,197 x$.

12. Sowhal, D.P.: Ilome monitoring and its role in the sudden infunt death syndrone. Pediatries $72: 133$, 1983.

13. Simpson, $H$ : Sudden unexpected death in infants: Home monitoring. Arch Dis 58: 469, 1983.

14. Peterson, D.R: Sudden unexpected death in infants: an epidemiologic: study. Am J Fidemiol $84: 478,1966$

15. Southall, D.P. Richards, J.M., Rhoden. K.J. et al. Prolonged apnoca and cardiac arrtythmies in infant discharges iton neoratal intensive care units: fuilure to podict on increased risk tor sudden intant death syndrome. Pediatries 70: 844, 1982.

16. Southall, D.P., Kichards, J.M., De Swtte, M. et al: lirst report of a multicentered prospective study into the sudden infant death syndrome: Identificntion of inlants dextined to die unexpectcally during infancy: Fivaluation of predictive inportante of prolonged apnea and aivorders of cardiac rytm or conduction. Br Mat J 286: 1092, 1983.

17. Mac Kay, M., Abreu, E., Silva, F.A et al: Home monitoring for central apnoca. Arcly Dis Child 59 136,1984 .

18. Peterson, D.R., Chinn, N.M., Fischer, I,D.: 'The sudden infant death syndrome: Repetitions in familics. J Pediatr 97: 265, 1980. 
19. Peterson, D.R., Sabotto, M.A., Daline, J.R.: ]nfants mortality among subsequent siblings of infant who died of sudden infant death syndrome. J Pediatr 108: $911,1986$.

20. Black, L., David, R., Broullete, R.T. et al: Fiffects of birth weight and etnicity in incidence of sudden infant death syndrome. J Pediatr 108: 209, 1986.

21. Hillman, L., Hoffmon, J.J., Hones, M. et al: Rclationship of prenatal and neonatal factor to SIDS:
Preliminary results of the NICHD cooperative epidemiological study of sudden infant deat syndrome (SIDS) rikk factors. Pediatr Res 16: 291, 1982 .

22. Trgens, L.M., Siljaeven, R., Peterson, Dr, et at: Prospective assessment of recutrence risk in sudden infant death syndrome. J Pediatr 104: 349, 1984.

23. Merrit, T.A., Valdes-Dapent, M.: SIDS Research Update. Pediatu Ann 13: 193, 1984. 